\title{
Compound heterozygous mutations of two elF2B genes in early childhood onset form of vanishing white matter disease
}

\section{Chunmei Wang}

Children's Hospital of Shanghai https://orcid.org/0000-0001-9350-7801

\section{Fang Yuan}

Children's Hospital of Shanghai

\section{Shengnan Wu}

Children's Hospital of Shanghai

Zhao Liu

Children's Hospital of Illinois

\section{Simei Wang}

Children's Hospital of Shanghai

\section{Xiaoping Lan}

Children's Hospital of Shanghai

\section{Quanmei Xu}

Children's Hospital of Shanghai

Xiaona Luo

Children's Hospital of Shanghai

\section{Yilin Wang}

Children's Hospital of Shanghai

\section{Wuhen Xu}

Children's Hospital of Shanghai

Jiaming Xi

Children's Hospital of Shanghai

\section{Yanfen Lu}

Children's Hospital of Shanghai

\section{Yuanfeng Zhang}

Children's Hospital of Shanghai

\section{Qin Lu}

Children's Hospital of Shanghai

\section{Jianjun Huang}

Children's Hospital of Shanghai

\section{Sreenivas K. Avula}


Children's Hospital of Illinois

\section{Abdullah Tolaymat}

Children's Hospital of Illinois

\section{Anqi Wang}

Children's Hospital of Shanghai

Jia Jia

Shanghai Center for Bioinformation Technology

Yucai Chen ( $\nabla$ chenyucaiphd@hotmail.com )

Children's Hospital of Shanghai

\section{Case report}

Keywords: vanishing white matter disease (VWMD), compound heterozygous mutations, novel mutation

Posted Date: March 9th, 2020

DOI: https://doi.org/10.21203/rs.3.rs-16411/v1

License: (c) (1) This work is licensed under a Creative Commons Attribution 4.0 International License. Read Full License 


\section{Abstract}

Background Diagnoses of vanishing white matter disease (VWMD) were difficult due to variable clinical features, severity, age of onset and wide range of mutations in elF2G genes which cause VWMD. This study reported two novel mutations in elF2B genes associated with VWMD to and expand our understanding of VWMD.

Case presentation Relevant data from clinical diagnoses and genetic mutational analyses in two Chinese female patients with sporadic VWMD were collected and analyzed. Protein structure/function was predicted. The identity of biological parents was confirmed based on variants called from the nextgeneration sequencing (NGS) data. Compound heterozygous mutations, c.254 T>A (p.Val85Glu), and c.597+2delT in the EIF2B2 gene, c.545C>T(p.Thr182Met) and c.1340C >T(p.Ser447Leu) in the EIF2B5 gene were detected in the two patients. Further phenotype investigation of both patients enables the diagnosis of the vanishing white matter disease. Three missense mutation c.254 T>A ( .Val85Glu) in the EIF2B2 gene, c.545C>T(p.Thr182Met) and c.1340C>T(p.Ser447Leu) in the EIF2B5 gene have been found and predicted to be deleterious. All the three mutation causes hydrophobicity and stability changes of proteins, and all the mutations were localized in conserved sequences. One novel mutation of c.1340C>T(p.Ser447Leu) in the EIF2B5 gene, and two other known mutations. The iterative threading assembly refinement (I-TASSER) server generated three-dimensional (3D) atomic models based on protein sequences from the novel missense mutation of c.1340C>T(p.Ser447Leu) in the EIF2B5 gene, which showed that the protein structure changed. The novel mutation c.597+2delT in the EIF2B2 gene may cause the splice site to disappear. We also analyzed mutations in missense mutations that cause VWMD and found that most of the t Pathogenic sites are localized in conserved NT and I-patch homology regions and the catalytic domain of EIF2BE.

Conclusions This study expands the spectrum of genotypes and phenotypes of VWMD and provides new insights into the molecular mechanism of VWMD and aide the acute diagnosis and treatment of VWMD.

\section{Background}

Vanishing white matter disease (VWMD, OMIM\#603896) is a rare neurologic disease inherited in an autosomal recessive manner. It has been proven that mutations in the genes encoding subunits of the eukaryotic translation initiation factor 2B are involved in the generation of VWMD [1]. The clinical phenotype of VWMD is closely linked to white matter demyelinating lesions and associated progressive neurologic deficits including spasticity, progressive cerebellar ataxia, epilepsy and cognitive impairment. Outside stress such as minor head trauma, infection with fever and acute fright might lead to rapid neurological deteriorations [2]. The onset of symptoms begins in young children but may appear at or shortly after birth or later in life. Typical onset of VWMD is between the ages of 2-6 years but varies from prenatal to adulthood. Patients with early-infantile onset, especially before the age of 2 years, present a severe phenotype that commonly leads to progressive deterioration and early death [3]. Brain MRI reveals extensive white matter abnormalities, with the signal intensity of the abnormal white matter close to that 
of cerebrospinal fluid [4,5]. VWMD is caused by mutations in any of the five genes: EIF2B1, EIF2B2, EIF2B3, EIF2B4, and EIF2B5 [6]. Due to the complex clinical phenotype of VWMD, it is often challenging in clinical practice for acute diagnosis of VWMD. The progress in molecular analysis technology has provided new tools to aid in accurate diagnosis of VWMD. Here, we present two patients with VWMD who were precisely diagnosed by the technique of exome sequencing in combination with brain MRI and clinical features. The results of this study provided new insights into the molecular mechanism of VWMD.

\section{Methods}

\subsection{Exome sequencing}

\subsubsection{DNA isolation}

The study program was approved by the Review Board of the Shanghai Children's Hospital of Jiaotong University, and the parents of the patients have signed the informed consent. Genomic DNA was isolated from peripheral blood using a QIAamp DNA Blood Midi Kit (Qiagen, Hilden, Germany) following the manufacturer's protocol.

2.1.2 Next-generation sequencing (NGS) and DNA sequence analysis

Genomic DNA samples were sheared by sonication. The sheared genomic DNA was then hybridized with the NimbleGen 2.0 probe sequence capture array from Roche

(http://www.nimblegen.com/products/seqcap/ez/v2/index.html) in order to enrich the exonic DNA (Joy Orient, China). The libraries were first tested for enrichment using qPCR and for size distribution and concentration using the Agilent Bioanalyzer 2100.

Data filtering, mapping and variant detection

The exon-enriched DNA was sequenced using the Illumina Hiseq2500 platform according to the manufacturer's instructions (Illumina). The raw image file was processed using BclToFastq (Illumina) for base calls to generate raw sequence data. A quality score of $\geq 20$ was used to filter out low quality variation. The sequencing read were compared with the NCBI human reference genome using BWA software. To analyze the single nucleotide variants (SNVs) and small insertions and deletions (InDels) of these two patients, we used the Samtools and Pindel software.

Data analysis

The data analysis was applied as follows:

- Synonymous changes and single nucleotide polymorphisms (SNPs) with a minor allele frequency higher than 5\% were removed (http://www.ncbi.nlm.nih.gov/projects/SNP).

- Nonsynonymous changes were filtered using the software SIFT (http://sift.jcvi.org). 
- Analysis was performed of the function of mutated genes and the association with the disease.

2.1.3 Mutation confirmed by Sanger method sequencing

The PCR product was sequenced using a ABI 3730XL and analyzed with DNASTAR software in order to confirm the mutations discovered in the patients

\subsection{Protein function prediction for the missense mutation}

To predict the effect of amino acid substitutions, we performed in silico analysis using the PROVEAN (http://sift.jcvi.org) and.The variant is predicted to be deleterious if the PROVEAN score is $\leq 2.5$, and neutral if the score is $>2.5$.

\subsection{Protein hydrophobicity analysis}

We use the software ProtScale (https://web.expasy.org/protscale/) and the software ProtParam (https://web.expasy.org/protparam/) to predict the hydrophobicity of the protein. The A Hphob. / Kyte \& Doolittle method was used to estimate the hydrophilicity index of each amino acid on the protein. The hydrophilicity of the protein was expressed by the Grand average of hydropathicity (GRAVY). The GRAVY less than 0 indicates hydrophilic protein, and the GRAVY s greater than O凶suggest a hydrophobic protein.

\subsection{Protein stability analysis}

To predict the stability of the protein after missense mutation, we use the software (http://mupro.proteomics.ics.uci.edu).The result is represented by the value detal delta G. If the stability is reduced, it is represented by DECREASE stability.

\subsection{Protein structure prediction on the I-TASSER server for the nonsense mutation and intron splicing mutation}

The I-TASSER Suite pipeline consists of 4 general steps:threading template identification, iterative structure assembly simulation, model selection and refinement, and structure-based function annotation. The server is available at囚http://zhanglab.ccmb.med. umich.edu/I-TASSER .

\subsection{Conserved sequence analysis}


Analysis 3 missense mutation sequences on alleles using the Clustal Omega software (http://www.ebi.ac.uk/Tools/msa/clustalo/).

\subsection{Ethical considerations}

This study was approved by the Ethics Committee of Shanghai Children's Hospital. Informed consent was obtained from the parents of the patient for using their blood samples for genetic analysis. The study complied with Chinese bioethics laws and the Helsinki declaration.

\section{Results}

\subsection{Clinical data पTable 10}

\section{Case A.}

In 2016, a 4-year-old Chinese girl, presenting with mild walking difficulty caused by minor head trauma was referred to the Neurology Department of the Shanghai Children's Hospital for treatment. She was born of nonconsanguineous parents at 39 weeks gestation with birth weight $3 \mathrm{~kg}$ and birth length $50 \mathrm{~cm}$. She was reported to by the parents be normal, except she had a mild mental development delay. Ataxia or spasticity had also been observed by her parents. Neither electroencephalogram test nor Electrocardiogram revealed abnormalities The results of the brain magnetic resonance imaging indicated extensive white matter damage, with major damage appearing around the area of the dentate nucleus and globus pallidus (Fig. 1a,b,c). No significant results were reported from screening for metabolic errors, including analyzing the amino acids level in the blood or urinary organic acid analysis. Family history is negative for genetic disorders and not contributory.

\section{Case B.}

In 2015, 3-year-old Chinese girl with mental retardation and recurrent epilepsy was admitted to the Shanghai Children's Hospital for treatment. She had recurrent seizures without fever onset at 7 months. Her seizures could be controlled by Levetiracetam combined with Oxcarbazepine, but occasionally occurred secondary dmage following infections. A psychomotor delay was observed by her parents in her first year of life. She had bilateral cataracts that occurred at the age of 31 month-old, which were successfully corrected by surgical operation. The electroencephalogram test showed diffuse non-specific abnormalities. The Electrocardiogram examination was normal. Liver function test was unremarkable with alanine aminotransferase at $26 \mathrm{U} / \mathrm{L}$ (normal range 5-40 U/L) and aspartate aminotransferase at 33 $\mathrm{U} \varangle \mathrm{L}$ (normal range 8-40 U/L). Her pulmonary, urological, cardiac, and renal functions and her levels of glucose and lactate were normal. She could walk with help, but her language ability was poor.

Magnetic resonance imaging (MRI) of the brain revealed that the symmetrical diffuse signal in the cerebral white matter was close to the cerebrospinal fluid on T1-weighted, T2-weighted and flair images 
(Fig. 1). No significant results were reported from screening for metabolic errors, including analyzing the amino acids level in the blood or urinary organic acid analysis. The girl was G2P1 with a birth weight of $3190 \mathrm{~g}$. No history of genetic disorder has been found in her family.

\subsection{Identification of Novel Mutations in EIF2B5 and EIF2B2}

In case A, two missense heterozygous variants were identified as compound heterozygous in the EIF2B5 gene, c. 545C>T(p.T182M) at exon 4 and c.1340C $>T$ (p.S447L) at exon 9, which were inherited from the mother and the father respectively (see Figure 3 for more detail). One missense heterozygous variant and one canonical splicing variant in the EIF2B2 gene, c.254 T>A (p.V85E) at exon 2 and c.597+2del T at exon 4 inherited from the parents, were found in the compound heterozygous state in case B (Fig. 2). Pedigree of the 2 sporadic patients (Fig. 3).

\subsection{Protein function prediction of the gene mutations}

The PROVEAN scores of the two missense mutations c.545C>T (p.T182M) and c.1340C >T (p.S447L) in EIF2B5 gene are -5.677 and -3.783 . The PROVEAN score of the missense mutations c.254 T>A (p.V85E) in EIF2B2 gene is -5.55 . Three missense mutation were predicted to be deleterious by the PROVEAN software. Missense mutations c.1340C>T(p.S447L) in EIF2B5 gene has not been reported yet.

\subsection{Protein hydrophobicity}

Estimation of the Hydropathic Index of Each Amino Acid on ALMS1 Protein by A Hphob. / Kyte \& Doolittle Method by ProtScale Software. The Grand average of hydropathicity of wild-type EIF2B5 protein was predicted by ProtParam software to be -0.276 . The Grand average of hydropathicity of the predicted protein after c.545C>T(p.T182M) mutation is -0.273 . The Grand average of hydropathicity of the predicted protein after $1340 \mathrm{C}>\mathrm{T}$ (p.S447L) mutation is -0.270 . It was found that $t$ both sites can cause a decrease in protein hydrophilicity after mutation. The Grand average of hydropathicity of wild-type EIF2B2 protein was predicted by ProtParam software to be -0.138 . The Grand average of hydropathicity of the predicted protein after c.254 T>A (p.V85E) mutation is -0.160 .

\subsection{Protein stability analysis}

The protein after c.545C>T(p.T182M) mutation in EIF2B5 predicted both value and sign of energy change using SVM and sequence information only (Recommended) delta delta $\mathrm{G}=-0.52567326$ (DECREASE stability). The protein after c.1340C>T (p.S447L) mutation in EIF2B5 predicted both value and sign of energy change using SVM and sequence information only (Recommended) detal delta $\mathrm{G}=-0.088498148$ (DECREASE stability). The protein after $254 \mathrm{~T}>\mathrm{A}$ (p.V85E) mutation in EIF2B2 predicted both value and sign of energy change using SVM and sequence information only (Recommended) detal delta $\mathrm{G}=$ -1.5715961 (DECREASE stability). The proteins after three missense mutations showed a decrease in stability predicted by 囚http://mupro.proteomics.ics.uci.edu.囚[7] 


\subsection{Protein structure prediction on the I-TASSER server for the missense mutation c.1340C>T(p.S447L) mutation in EIF2B5}

The ITASSER server generated vastly different 3D atomic models based on protein sequences from widetype and novel missense mutation of c.1340C>T (p.S447L) mutation in EIF2B5 (Figs. 4 and 5)

\subsection{Conserved sequence analysis}

After analysis with the Clustal Omega software. Assembly showed that the locations of mutations on both alleles were in conserved sequences. Conservation analysis shows all three missense variants were highly conserved in species, which indicates these changes could not be tolerated. (Figs.6)

\section{Discussion}

VWM is a rare genetic disease that is classified in five subtypes depending on the onset age: 1) the prenatal/congenital form with onset before birth, 2) the subacute infantile form with onset occurring at less than 1 year of age, 3 ) the early childhood onset form with onset occurring between 1-5 years, 4) the late childhood /juvenile onset form with onset occurring between 5-15 years, and 5) the adult onset form with onset beginning at 15 years old [8]. The major feature of VWMD is that patients may be normal or slightly delayed at the beginning and then symptoms worsen slowly, but rapid deterioration can occur because of infection, head trauma, and other acute psychological stresses. The main phenotypes of VWMD are ataxia, spasticity, and epilepsy in some patients [9]. Progressive rarefaction and cystic degeneration occurring in the white matter of the brain are the typical features of VWMD. Magnetic resonance imaging (MRI) of the brain shows symmetrically and diffusely abnormal white matter, with the signal intensity in the abnormal white matter is close to that of cerebrospinal fluid on T1-weighted, T2weighted and fluid-attenuated inversion recovery [5]. Eline M. C et al [10]. performed a longitudinal multicenter study of 296 genetically diagnosed VWM patients and found that the median age of the first onset was 3 years old, with $60 \%$ of patients developing symptoms before the age of 4 years old. The nature of the first signs varied for different ages of onset. Overall, motor problems were the most common presenting sign, especially in children. Adolescent and adult-onset patients are more likely to show cognitive problems early after disease onset. The earlier the onset age, the higher the mortality rate, especially the age of onset is less than 1 year old. Absence of stress-provoked episodes and absence of seizures predicted more favorable outcome. In our study, the age of onset of patient B was 7 months, and combined with epilepsy and difficulty walking. The age of onset of patient A was 4 years old, no seizures, clinical symptoms were lighter than patient $\mathrm{B}$.

VWM can be related to mutations in any of the five genes (EIF2B1-5), encoding the five subunits of eukaryotic translation initiation factor elF2B (elF2Ba, $\beta, \gamma, \delta$, and $\varepsilon$ ). elF2B assumes a task in the initiation of mRNA translation. Under stress conditions, protein synthesis is inhibited via elF2B inactivation [11]. EIF2B5 is the most frequently mutated gene; mutations in EIF2B5 are detected in $57 \%$ of the patients. Mutation rates of the other genes are EIF2B4 (17\%), EIF2B2 (15\%), EIF2B3 (7\%), and EIF2B1 (4\%) respectively [12]. The domain structure of EIF2B $\varepsilon$ showing the conserved NT (aa 44-165) and I-patch 
homology regions (aa 347-437) and the catalytic domain (Cat) with approximate residue numbering (aa 547-721). NTs is a conserved domain with sequence similarity to nucleotidyl transferases. which are highly conserved throughout eukaryotes, play key roles in the interactions between subunits in the elF2B complex. I-patch regions are important in the interactions between the catalytic elF2B $\varepsilon$ complex and the other subunits. Cat enhanced by the presence of the other subunits [13]. Since Leegwater PA et al [14] first discovered that the EIF2B5 mutation caused VWMD, more EIF2B5 mutations that caused VWMD have been discovered. We summarized NCBI Reference Sequence NM_003907.3(EIF2B5) about 61 site mutations together with our report, 41 of which are missense mutations. Associated with

Leukoencephalopathy with vanishing white matter are 27 missense mutations. Moreover, these missense mutations are mainly distributed in exons 1, 2, 4, 8. Among these 27 missense mutations, fourteen were marked pathogenic or likely pathogenic in the ClinVar Database囚Table 2Х. Passemard S, et al [15]. studied four patients, two patients were compound heterozygotes (p.R315H/p.F56C). One became comatose during a viral infection at age 18 months and died and another one died at age 8.5 with an impressive macrocephaly. Maletkovic J,etal [16] reported 9 novel mutations in the EIF2B genes, Among them, c. $241 \mathrm{G}>\mathrm{A}$ (p.Glu81 Lys) is related to VWMD and is pathogenic. The mutations in EIF2B5 gene were associated with both mild and severe phenotypes. Gonca Bektaşa,etal[17] reprted patients homozygous for p.Arg113His, in terms of age at onset and age at loss of independent walking. We analyzed EIF2B5 gene missense mutations and found that $8 / 14$ is distributed in I-patch domain,Cat domain,NTdomain, which three functionally important areas (Fig.7). We summarized NM_014239.4 (EIF2B2) about 35 site mutations, 13 of which are missense mutations. Associated with Leukoencephalopathy with vanishing white matter are 7 missense mutations,only five were marked pathogenic or likely pathogenic in the ClinVar Database $₫$ Table 2\.

We utilized trio-based WES in two cases affected with VWMD and their parents to investigate underlying genetic causes of this disease. We identified two compound heterozygous mutations in EIF2B5 and EIF2B2 in each patient. Mutations in one of the subunits of the eukaryotic translation initiation factor genes, named EIF2B may lead to similar clinical phenotypes[18].The mutation of these genes results in reduced function of elF2B, which is a key issue during stresses such as fever, infection, or head trauma[19].In case A, the onset began at 4 years old. She has only mild walking problems, and MR imaging marked the typical brain white matter damage of VWMD. Further genetic testing proved the clinical diagnosis, showing a heterozygous mutation in the EIF2B5 gene: c.545C>T(p.T182M) in EXON 4, which was inherited from her mother, and c.1340C>T(p.S447L) in EXON 9, which was inherited from her father. In case $B$, the onset began at 7 months and should be classified as the subacute infantile form. The compound mutation in EIF2B2 in this case was c.254 T>A (p.v85E) in EXON 2, which was inherited from her father, and c.597+2del T in EXON4, which was inherited from her mother. Three missense mutation were predicted to be deleterious by the PROVEAN software. Missense mutations c.1340C>T(p.S447L) in EIF2B5 gene has not been reported yet. Missense mutations c.545C>T(p.T182M) in the EIF2B5 and missense mutations c.254 T>A (p.v85E) in the EIF2B has been reported [20-21]『but the two missense mutations are all adults, Homozygous mutation and the clinical symptoms are lighter. The two children we reported the age of onset was earlier, with a 4-year-old onset, a 7-month-old onset, severe 
clinical symptoms, and a compound heterozygous mutation. Through hydrophobic analysis and protein stability prediction, it was found that missense mutations at three sites can cause changes in the hydrophobicity of proteins, decrease the stability of proteins, and affect the transport function of proteins. The conservation analysis showed that all the missense mutations occurred at conserved positions. The ITASSER server generated vastly different 3D atomic models based on protein sequences from wide-type and novel missense mutation of c.1340C > T(p.S447L) mutation in EIF2B5. We notice that case $B$ has a more severe clinical phenotype; the deletion c.597+2del T in Intron 4 occurred in this patient, which might lead to disappearance of the original splice site. splicing mutations, causing changes in protein structure. It would be better to confirm this using RT-PCR, but unfortunately, the parents of the patient declined to conduct further investigation.

Since the mutations come from different genes, it is difficult to compare the differences in clinical phenotype. It is interesting to see that case A carries a mutation that will result in a truncated protein. Additionally, case B also has cataracts, which suggests that VWMD phenotypes are not limited to brain damage. According to publications, stress in the endoplasmic reticulum and UPR activation might be the etiology of cataracts.

Identifying the genetic basis of VWMD in these two patients allows us to provide genetic counseling for their families and give them the option of prenatal testing, as in patient $B$ whose mother has delivered a normal boy after prenatal testing. The neurological deterioration of these two patients was caused by febrile infections and minor head trauma.

There is no cure for this devasting disease. Physical therapy and rehabilitation for motor dysfunction and antiepileptic drugs for seizures control are suggested in clinical practice. In addition, to avoid possible deterioration from outside attack, patients are advised to for vaccinations, low-dose of maintenance antibiotics during winter, antibiotics for minor infections, antipyretics for fever, and helmet when outside to minimize the impact of brain trauma. But scientists have been searching for ways to treat this disease. Zhou $L$ et al [22] fibroblasted from two VWMD children were reprogrammed into iPSCs by using a virusfree nonintegrating episomal vector system. They discovered the astrocyte abnormalities, whether it can inhibit astrocyte abnormalities to treat VWMD, provides us with a new perspective. Mutations in elF2B genes cause VWMD, a fatal leukodystrophy that can manifest following physical trauma or illness, conditions that activate the integrated stress response (ISR) [23]. Hodgson RE et al [24]. provided evidence that the composition and function of mammalian elF2B bodies are regulated by the ISR and the drugs that control it. Abbink TEM et al [25] found the compound ISRIB (for "ISR inhibitor") was administered to VWMD mice to increase elF2B activity. In brains of VWMD compared to wild-type mice we observed the most prominent changes in translation concerning ISR mRNAs,their expression levels correlated with disease severity.

\section{Conclusion}


We have reported here two VWMD cases with compound heterozygous mutations, c.254 T > A (p.Val85Glu), and c.597 + 2delT in the EIF2B2 gene, c.545C > T(p.Thr182Met) and c.1340C > $\mathrm{T}$ (p.Ser447Leu) in the EIF2B5 gene. The mutations c.597 + 2delT in the EIF2B2 gene and c.1340C > $\mathrm{T}$ (p.Ser447Leu) in the EIF2B5 gene are two novel mutations. Three missense mutation predicted to be deleterious. The iterative threading assembly refinement (I-TASSER) server generated three-dimensional (3D) atomic models based on protein sequences from the novel missense mutation of c.1340C > $T$ (p.Ser447Leu) in the EIF2B5 gene, which showed that the protein structure changed. The novel mutation c.597 + 2delT in the EIF2B2 gene may cause the splice site to disappear. This study expands the spectrum of genotypes and phenotypes of VWMD, provides new insights into the molecular mechanism of VWMD, and helps the diagnosis and treatment of VWMD.

\section{Abbreviations}

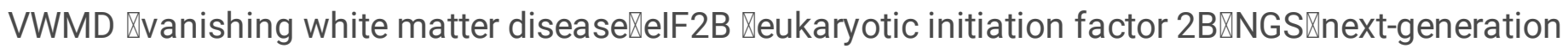
sequencing $\triangle T A S S E R \bigotimes T$ The iterative threading assembly refinement $\triangle M R I \otimes$ Magnetic resonance imaging $\bigotimes$

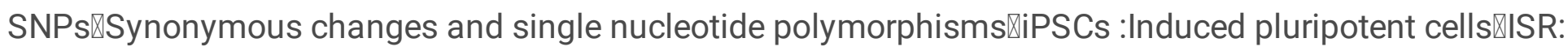
integrated stress response

\section{Declarations}

\section{Ethics approval and consent to participate}

The study procedure was permitted by the Ethics Committee of Children's Hospital of Shanghai, Shanghai Jiao Tong University,and informed written consent was obtained from the patient's guardian.

\section{Consent for publication}

A signed informed consent was obtained from the patient's guardian for publication of this case report and accompanying neuroimages.

\section{Availability of data and materials}

The datasets used and analysed during the current study are available from the corresponding author on reasonable request.

\section{Competing interests}

The authors declare that they have no competing interest. 


\section{Funding}

This work was supported by grants from the National Natural Science Foundation of China (No.81650008), Shanghai Science and Technology Fund (No.16410723400), Talent Introduction Fund of Shanghai Children's Hospital (to YC), Key Subject Program from Shanghai Municipal Commission of Health and Family Planning (No.2016ZB0102), Key disciplines of top priority in Shanghai (2017ZZ02019) and Shanghai Hospital Development Center (SHDC12015113).

\section{Authors' contributions}

$\mathrm{YC}, \mathrm{CW}$ and $\mathrm{FY}$ determined the study design and performed the study. QX, XL, YW, JX, YL, YZ,QL and JH were responsible for data collection. SW, WX, ZL, SW, XL, SA, AT,AW and JJ analyzed the data. CW drafted the manuscript. All authors read and approved the final manuscript.

\section{Acknowledgments}

We would like to express our gratitude to the patient and their parents for their support.

\section{References}

[1]Bugiani M, Vuong C, Breur M, van der Knaap MS. Vanishing white matter: a leukodystrophy due to astrocytic dysfunction. Brain Pathol. 2018 ;28(3):408-421.

[2]van der Knaap MS, Pronk JC, Scheper GC. Vanishing white matter disease. Lancet Neuro 2006;5(5):413-423.

[3] Fogli A, Schiffmann R, Bertini E, Ughetto S, Combes P, Eymard-Pierre E,

et al. The effect of genotype on the natural history of elF2B-related leukodystrophies. Neurology 2004;62:1509-17.

[4] Ding XQ , Bley A, Ohlenbusch AđKohlschütter A囚Fiehler J. Imaging evidence of early brain tissue degeneration in patients with vanishing white matter disease: a multimodal MR study. J Magn Reson Imaging 2012;35(4):926-32.

[5] van der Lei HD, Steenweg ME, Barkhof F, de Grauw T, d'Hooghe M, Morton R, et al. Characteristics of early MRI in children and adolescents with vanishing white matter.Neuropediatrics 2012;43(1):22-6.

[6]Takano K, Tsuyusaki Y, Sato M, Takagi M, Anzai R, Okuda M, 
et al.A Japanese girl with an early-infantile onset vanishing white matter disease resembling Cree leukoencephalopathy. Brain Dev 2015;37(6):638-42

[7] Cheng J, Randall A, Baldi P. Prediction of Protein Stability Changes for Single-Site Mutations Using Support Vector Machines. Proteins, 2006;62(4):1125-1132.

[8] van der Knaap MS, Fogli A, Boespflug-Tanguy O, Abbink TEM, Schiffmann R.

In: Adam MP, Ardinger HH, Pagon RA, Wallace SE, Bean LJH, Stephens K, Amemiya A, editors. GeneReviews ${ }^{\circledR}$ [Internet]. Seattle (WA): University of Washington, Seattle; 1993-2020.

[9] Bugiani M, Vuong C, Breur M, van der Knaap MS. Vanishing white matter: a leukodystrophy due to astrocytic dysfunction. Brain Pathol. 2018 ;28(3):408-421.

[10] Hamilton EMC, van der Lei HDW, Vermeulen G, Gerver JAM, Lourenço CM, Naidu S, etal. Natural History of Vanishing White Matter. Ann Neurol. 2018 ;84(2):274-288.

[11] Güngör G, Güngör O, Çakmaklı S, Maraş Genç H, İnce H, Yeşil G,et al. Vanishing white matter disease with different faces. Childs Nerv Syst. 2019; Aug 5.

[12] Turón-Viñas E, Pineda M, Cusí V, López-Laso E, Del Pozo RL, Gutiérrez-Solana LG, et al. Vanishing white matter disease in a spanish population. J Cent Nerv Syst Dis. 2014 Jul 13;6:59-68.

[13] Wang X, Wortham NC, Liu R, Proud CG. Identification of residues that underpin interactions within the eukaryotic initiation factor (elF2) 2B complex. J Biol Chem. 2012 Mar 9;287(11):8263-74.

[14] Leegwater PA, Vermeulen G, Könst AA, Naidu S, Mulders J, Visser A,et al. Subunits of the translation initiation factor elF2B are mutant in leukoencephalopathy with vanishing white matter. Nat Genet. 2001; 29(4):383-8.

[15] Passemard S, Gelot A, Fogli A, N'Guyen S, Barnerias C, Niel F,et al. Progressive megalencephaly due to specific EIF2Bepsilon mutations in two unrelated families.

Neurology. 2007 Jul 24;69(4):400-2.

[16] Maletkovic J, Schiffmann R, Gorospe JR, Gordon ES, Mintz M,et al. Genetic and clinical heterogeneity in elF2B-related disorder. J Child Neurol. 2008 ;23(2):205-15.

[17] Gonca Bektaşa, Gözde Yeşilb, Melis Ulak Özkana, Edibe Pembegül Yıldıza, Tuğçe Aksu Uzunhana, Vanishing white matter disease with a novel EIF2B5 mutation: A 10-year follow-up. Clin Neurol Neurosurg. 2018;171:190-193.

[18]Scali O, Di Perri C, Federico A.The spectrum of mutations for the diagnosis of vanishing white matter disease. Neurol Sci .2006;27(4):271-7. 
[19]Sharma S, Ajij M, Singh V, Aneja S. Vanishing white matter disease with mutations in EIF2B5 gene,Indian J Pediatr .2015;82(1):93-5.

[20] Matsukawa T, Wang X, Liu R, Wortham NC, Onuki Y, Kubota A,et al. Adult-onset leukoencephalopathies with vanishing white matter with novel missense mutations in EIF2B2, EIF2B3, and EIF2B5. Neurogenetics. 2011;12(3):259-61.

[21] Ohtake H, Shimohata T, Terajima K, Kimura T, Jo R, Kaseda R, et al.Adult-onset leukoencephalopathy with vanishing white matter with a missense mutation in EIF2B5. Neurology. $2004 ; 11 ; 62(9): 1601-3$.

[22]Zhou L, Li P, Chen N, Dai LF, Gao K, Liu YN,etal. Modeling vanishing white matter disease with patientderived induced pluripotent stem cells reveals astrocytic dysfunction. CNS Neurosci Ther. 2019;25(6):759771.

[23]Moon SL, Parker R. EIF2B2 mutations in vanishing white matter disease hypersuppress translation and delay recovery during the integrated stress response. RNA. 2018;24(6):841-852.

[24] Hodgson RE, Varanda BA, Ashe MP, Allen KE, Campbell SG. Cellular elF2B subunit localization: implications for the integrated stress response and its control by small molecule drugs .Mol Biol Cell. $2019 ; 30(8): 942-958$.

[25] Abbink TEM, Wisse LE, Jaku E, Thiecke MJ, Voltolini-González D, Fritsen H,et al. Vanishing white matter: deregulated integrated stress response as therapy target. Ann Clin Transl Neurol. 2019 Aug;6(8):1407-1422.

\section{Tables}

Table 1. Clinical features of the 2 patients. 
Sex

Female

Female

\begin{tabular}{|c|c|c|}
\hline Gene mutation & $\begin{array}{l}\text { c.545C }>\mathrm{T}(\mathrm{p} . \mathrm{Th} 182 \mathrm{Met}) \text { and } \\
\text { c.1340C }>\mathrm{T}(\mathrm{p} . \text { Ser447Leu }) \text { in the } \\
\text { EIF2B5 gene }\end{array}$ & $\begin{array}{l}\text { c. } 254 \text { T>A (p.Valo5Glu), and c.597+2delT in } \\
\text { the EIF2B2 gene }\end{array}$ \\
\hline Family history & No & No \\
\hline Clinical manifestation & $\begin{array}{l}\text { Spasticity, progressive cerebellar } \\
\text { ataxia, cognitive impairment. }\end{array}$ & $\begin{array}{l}\text { Spasticity,progressive cerebellar ataxia, not } \\
\text { walk alone@epilepsy and cognitive impairment }\end{array}$ \\
\hline $\begin{array}{l}\text { Accompanying signs } \\
\text { and symptoms }\end{array}$ & No & bilateral cataracts \\
\hline Age of onset & 4-year-old & 7 months \\
\hline $\begin{array}{l}\text { Reason for seeing a } \\
\text { doctor }\end{array}$ & $\begin{array}{l}\text { mild walking difficulty caused by } \\
\text { minor head trauma }\end{array}$ & mental retardation and recurrent epilepsy \\
\hline Electroencephalogram & Normal & diffusenon-specific abnormalities \\
\hline Brain MRI & Abnormal & Abnormal \\
\hline
\end{tabular}

Table 2. Analysis of missense mutations in EIF2B5 gene and EIF2B2 gene caused by Vanishing white matter disease 


\begin{tabular}{|c|c|c|c|c|c|}
\hline EIF2B5 & Exon 1 & NM_003907.3:c.115C>G & p.Pro39Ala & Uncertain & VCV000344329 \\
\hline EIF2B5 & Exon 1 & NM_003907.3:c.166T>G & p.Phe56Val & Pathogenic & VCV000005951 \\
\hline EIF 2B5 & Exon 1 & NM_003907.3:c.167T>G & p.Phe56Cys & Pathogenic & VCV000005952 \\
\hline EIF2B5 & Exon 1 & NM_003907.3:c.182A>G & p.Lys61Arg & Uncertain & VCV000344330 \\
\hline EIF2B5 & Exon 2 & NM_003907.3:c.271A>G & p.Thr91Ala & Pathogenic & VCV000005942 \\
\hline EIF 2B5 & Exon 2 & NM_003907.3:c.299C>T & p.Ala100Val & Uncertain & VCV000344332 \\
\hline EIF2B5 & Exon 2 & NM_003907.3:c.318A>T & p.Leu106Phe & Conflicting & VCV000195203 \\
\hline EIF2B5 & Exon 2 & NM_003907.3:c.338G>A & p.Arg 113 His & Pathogenic & VCV000005945 \\
\hline EIF2B5 & Exon 2 & NM_003907.3:c.349C> & p.Leu117Val & Uncertain & VCV000560268 \\
\hline EIF2B5 & Exon 4 & NM_003907.3:c.545C>T & p.Thr182Met & pathogenic & VCV000005949 \\
\hline EIF2B5 & Exon 4 & NM_003907.3:c.584G>A & p.Arg195His & Pathogenic & VCV000005946 \\
\hline EIF2B5 & Exon 4 & NM_003907.3:c.808G>C & p.Asp $270 \mathrm{His}$ & Pathogenic & VCV000040178 \\
\hline EIF2B5 & Exon 7 & NM_003907.3:c.925G>C & p.Val309Leu & Pathogenic & VCV000005947 \\
\hline EIF2B5 & Exon 7 & NM_003907.3:c.944G>A & p.Arg $315 \mathrm{His}$ & Pathogenic & VCV000598970 \\
\hline EIF 2B5 & Exon 7 & NM_003907.3:c.1010A>G & p.His337Arg & pathogenic & VCV000590971 \\
\hline EIF2B5 & Exon 8 & NM_003907.3:c.1157G>T & p.Gly386Val & Pathogenic & VCV000005944 \\
\hline EIF2B5 & Exon 8 & NM_003907.3:c.1159G>C & p.Asp387His & Uncertain & VCV000344334 \\
\hline EIF2B5 & Exon 8 & NM_003907.3:c.1265G>A & p.Arg $422 \mathrm{Gln}$ & Uncertain & VCV000560269 \\
\hline EIF2B5 & Exon 8 & NM_003907.3:c.1268T>C & p.Val423Ala & Uncertain & VCV000561000 \\
\hline EIF2B5 & Exon 10 & NM_003907.3:c.1529T>G & p.Leu510Arg & Uncertain & VCV000344336 \\
\hline EIF2B5 & Exon 11 & NM_003907.3:c.1621C>T & p.Arg 541Trp & Uncertain & VCV000344337 \\
\hline EIF2B5 & Exon 13 & NM_003907.3:c.1759A>G & p.Ile 587Val & Benign & VCV000094381 \\
\hline EIF2B5 & Exon 13 & NM_003907.3:c.1882T>C & p.Trp628Arg & Pathogenic & VCV000005943 \\
\hline EIF2B5 & Exon 14 & NM_003907.3:c.1946T>C & p.Ile649Thr & Pathogenic & VCV000420049 \\
\hline EIF2B5 & Exon 15 & NM_003907.3:с.2002A>G & p.Met668Val & Uncertain & VCV000344338 \\
\hline EIF2B2 & Exon 2 & NM_014239.4:c.254T>A & p.Val85Glu & Pathogenic & VCV000040180 \\
\hline EIF2B2 & Exon 2 & NM_014239.4:c.271G >C & p.Glu91Gln & Uncertain & VCV000314330 \\
\hline EIF2B2 & Exon 4 & NM_014239.4:c.570T>G & p.Ile190Met & Uncertain & VCV000314334 \\
\hline EIF2B2 & Exon 5 & NM_014239.4:c.599G>T & p.Gly200Val & Pathogenic & VCV000208575 \\
\hline EIF2B2 & Exon 5 & NM_014239.4:c.638A>G & p.Glu213Gly & Pathogenic & VCV000004336 \\
\hline EIF2B2 & Exon 8 & NM_014239.4:c.922G>A & p.Val308Met & Pathogenic & VCV000522641 \\
\hline EIF2B2 & Exon 8 & NM_014239.4:c.947T>A & p.Val316Asp & Pathogenic & VCV000004337 \\
\hline
\end{tabular}

\section{Figures}




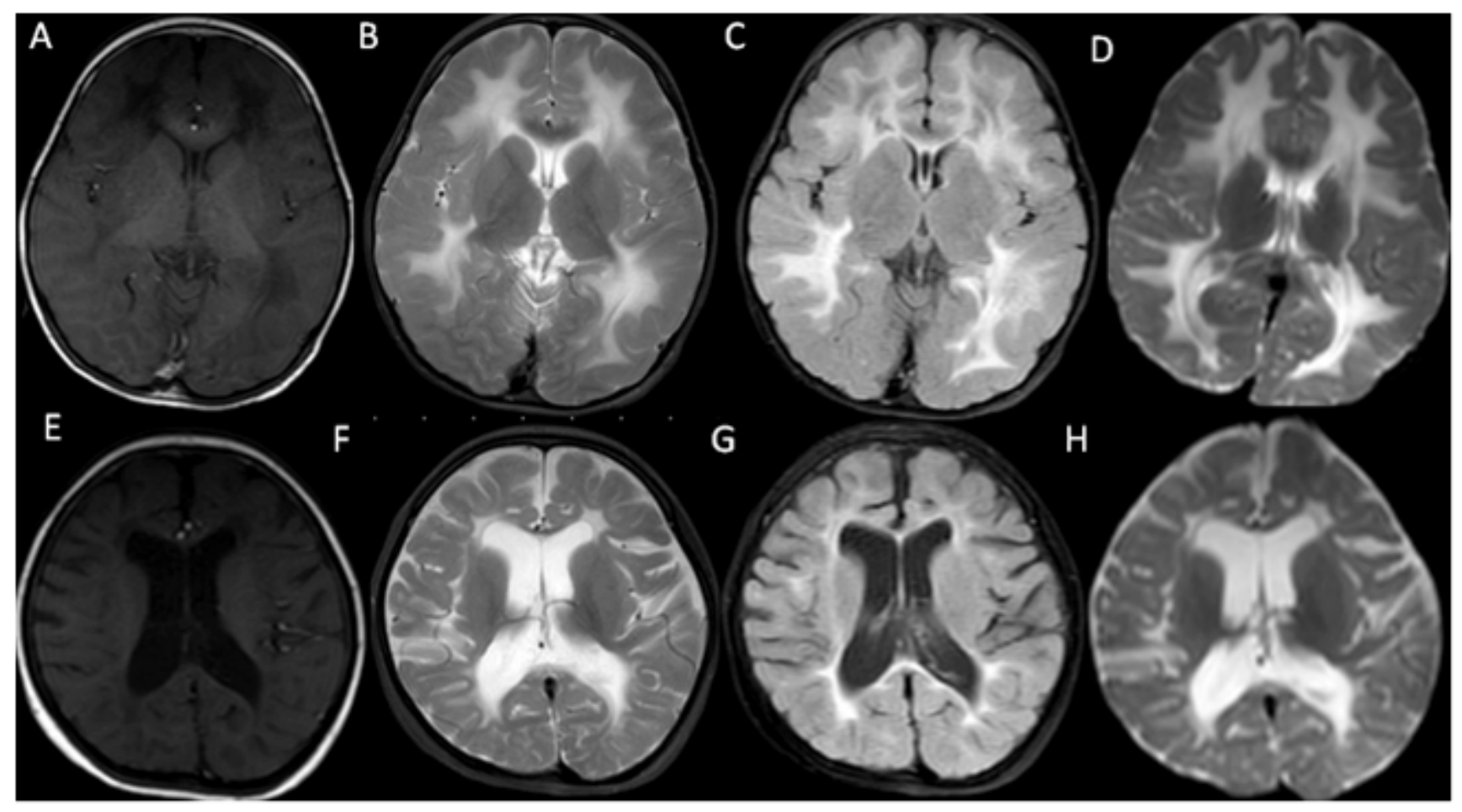

\section{Figure 1}

MRI showing generalized brain damage VWMFD.The brain MRI of Case $A(A, B, C, D)$ and Case $B(E, F, G$, $H)$, which show diffuse brain white matter damage on T1 weighted sequence imaging (A, E), T2 T1 weighted sequence imaging $(B, F)$, FLAIR images $(C, G)$ and DWI images $(D, H)$. 
c.545C >T (p.T182M)

Control

ATGTTTCTGTGATGACGATGATCTTCA A GG

Patient

A TGTTTCTGTGATGATGATGATCTTCA A G G

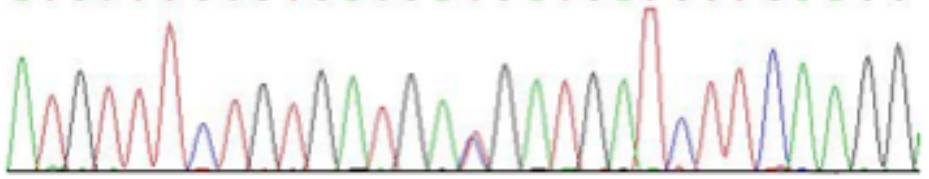

Father

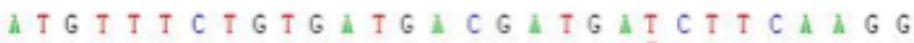

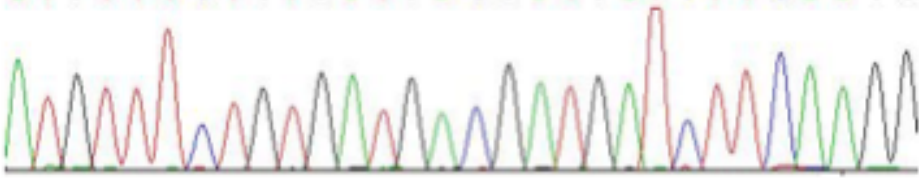

Mother

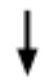

A TGT T TC TG TG A TG A TGATGATCTTCA A G G

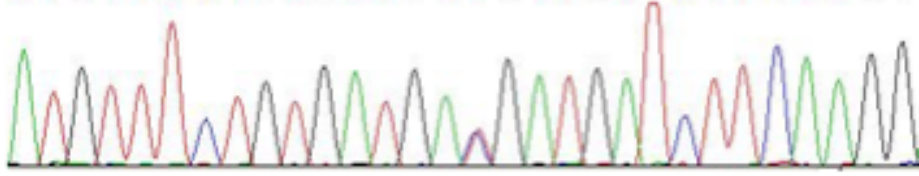

c.1340C $>T$ (p.S447L)

Control

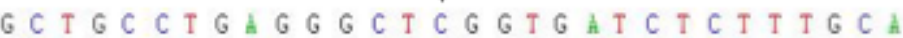

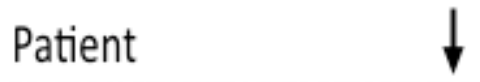

GCTGCCTGAGGGCTTGGTGATCTCTTTGCA
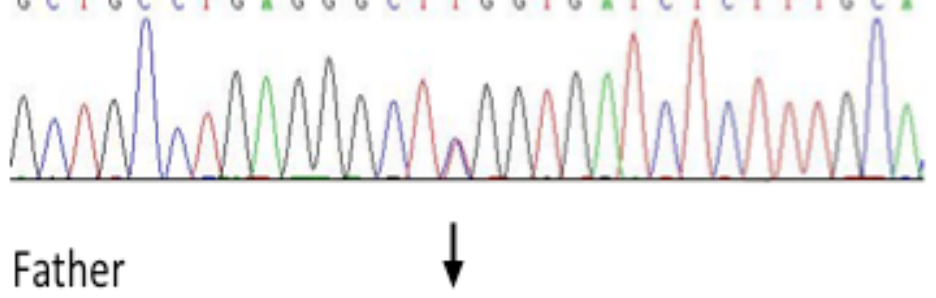

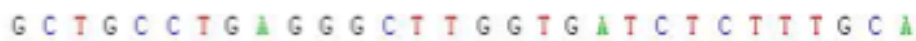
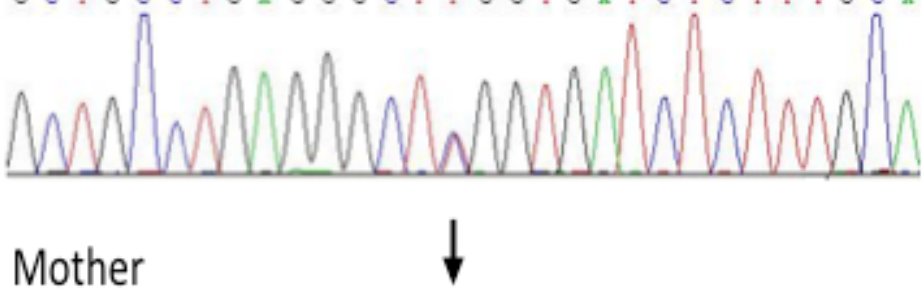

GCTGCC TGAGGGCTCGGTGATCTCTITGCA

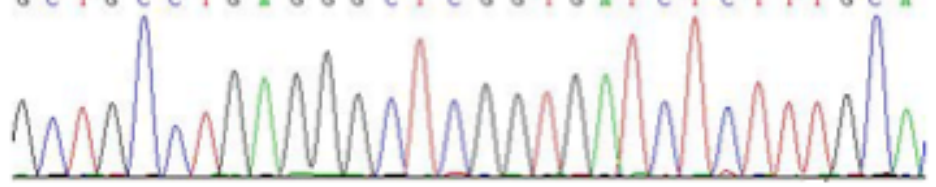

Figure 2

Gene mutations in Case A shown using the $A B I 3730 X L$.Sanger sequencing results on the position of c.545C>T(p.T182M) and c.1340C>T(p.S447L) of EIF2B5 gene. Electropherograms showing the DNA sequence at the position of EIF2B5 gene c.545C>T(p.T182M) in this patient (top). Her mother carried a $\mathrm{C}>\mathrm{T}$ in this position (bottom), whereas her father was normal (middle). Electropherograms showing the DNA sequence at the position of EIF2B5 gene c.1340C >T(p.S447L) in this patient (top). Her father carried a $\mathrm{C}>\mathrm{T}$ in this position (middle), whereas her mother was normal (bottom). 
Patient A EIF2B5

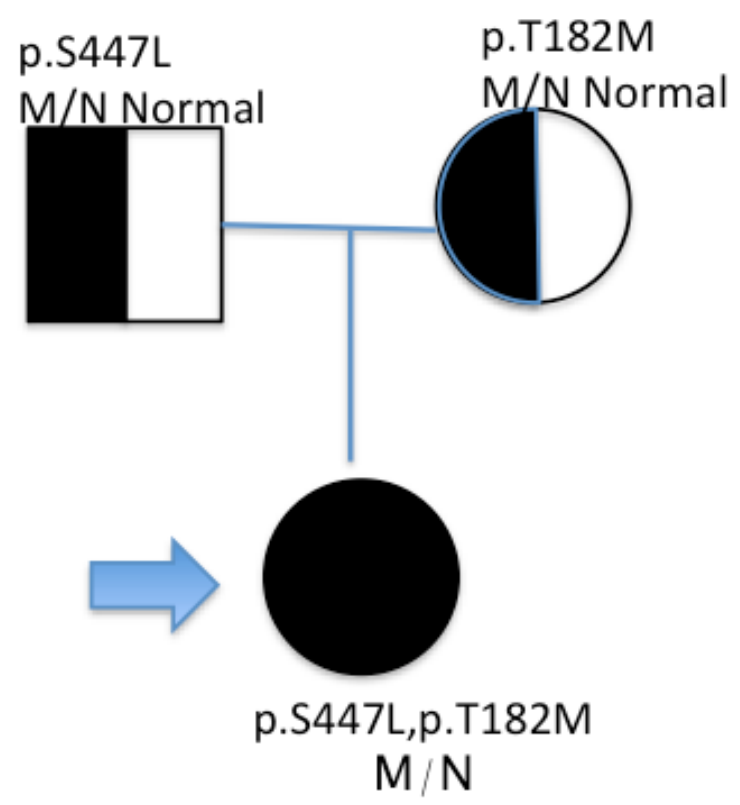

Patient B EIF2B2

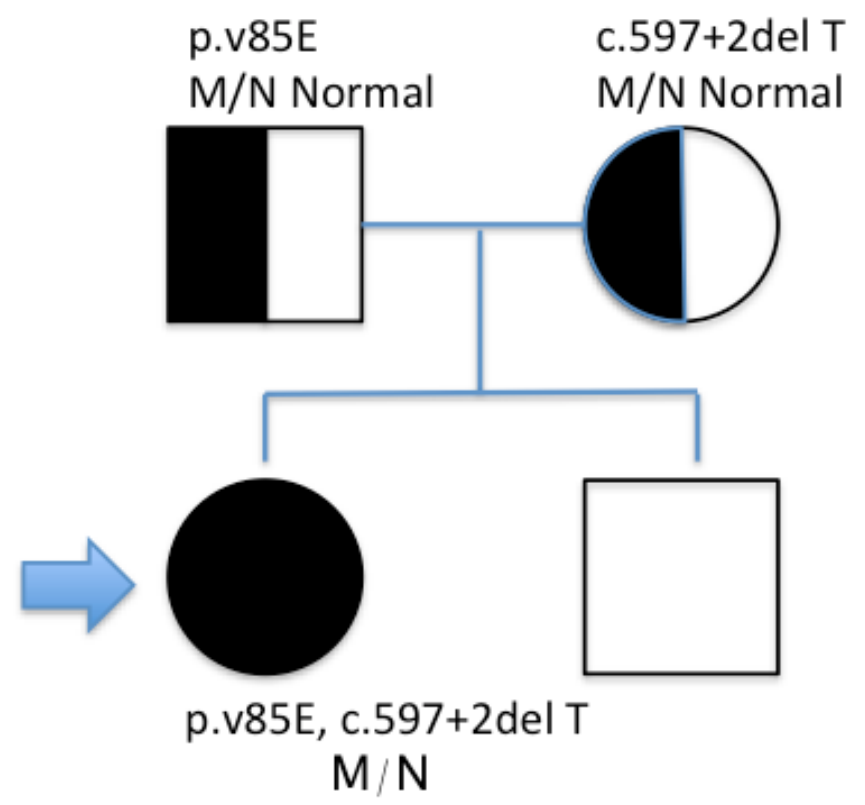

Figure 3

Pedigree of the 2 sporadic patients. 


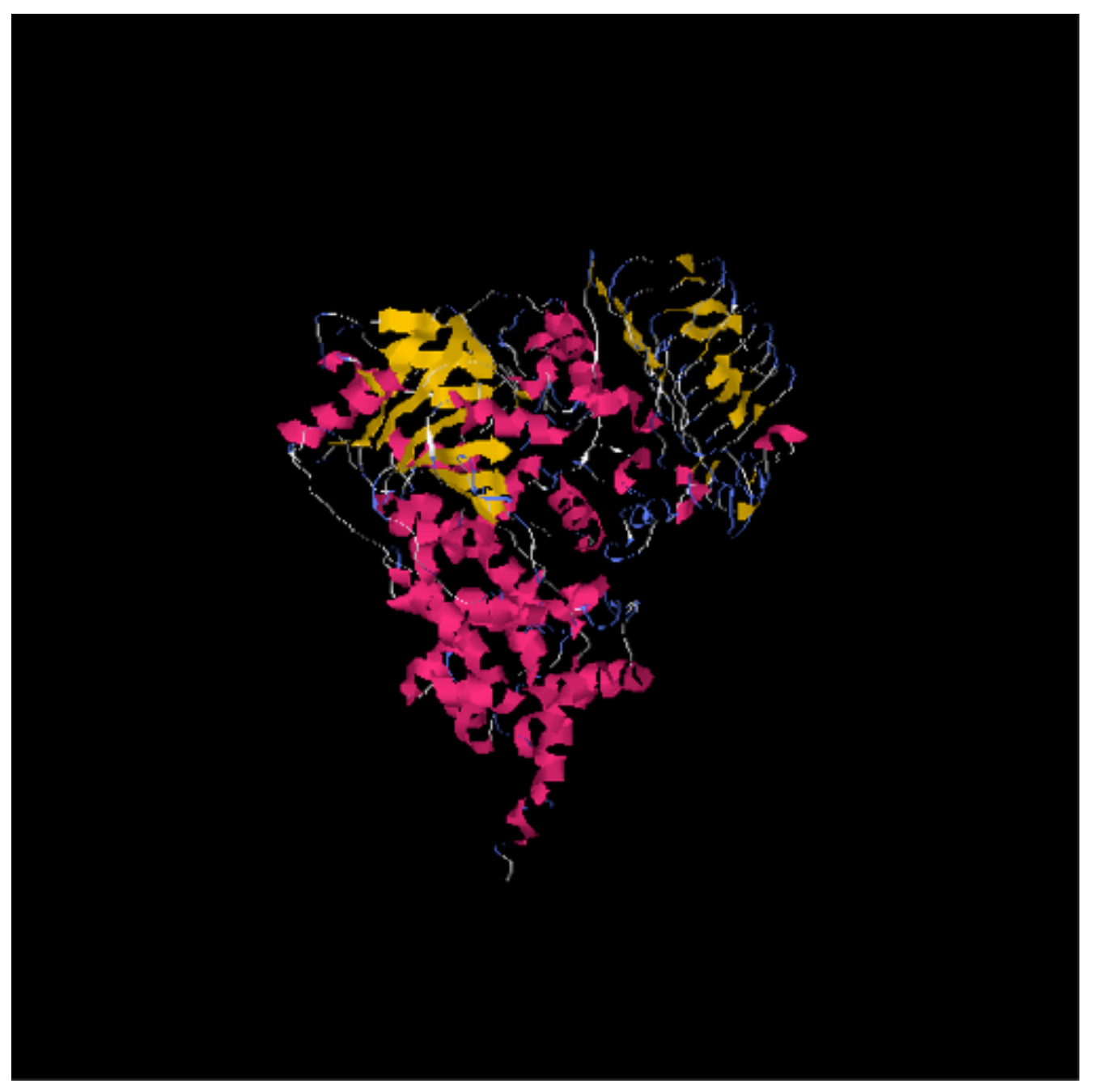

\section{Figure 4}

The 3-dimensional atomic model based on protein sequence of EIF2B5 from wide-type generated by iterative threading assembly refinement server. 


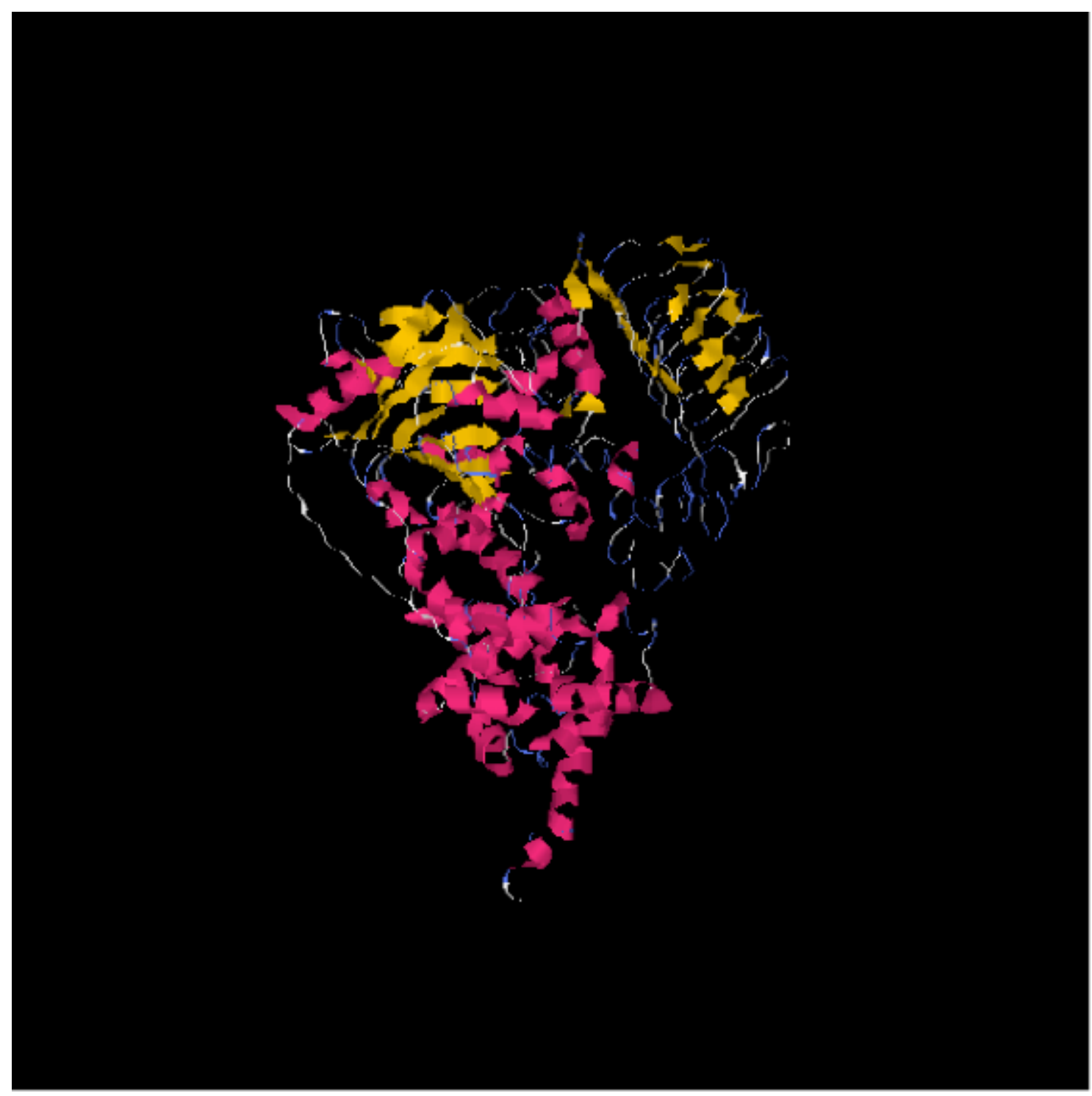

\section{Figure 5}

The 3-dimensional atomic model based on protein sequence from the missense mutation of c.1340C > T (p.S447L) in EIF2B5 generated by the iterative threading assembly refinement server. 


Rattus
Mus
Oryctolagus
Macaca
Homo
Pan
Bos
Canis

Rattus
Mus
Oryctolagus
Macaca
Homo
Pan
Bos
Canis

\section{ElF2B5 gene :T182M}

$\checkmark$

LEKNVSVMTMVEKESSPSHPTRCHEDNVVLAVDSTTNRILHFQKTQGLRHFSFPLGLFQG LEKNVSVMTMVEKESSPSHPTRCHEDNVVMAVDSATNRVLHFQKTQGLRRFSFPLSLFQG LEKNVSVMTMI EKESSPSHPTRCHEDNVVVAVDSATNRILHFQKTQGLRRFSFPLSLFQG LEKIVSVMTMI EKESSPSHPTRCHEENVVVAVDNATNRVLHFQKTQGLRRFSFPLSLFQG LEKNVSVMTMI FKESSPSHPTRCHEDNVVVAVDSTTNRVLHFQKTQGLRRFAFPLSLFQG LEKNVSVMTMI FKESSPSHPTRCHEDNVVVAVDSTTNRVLHFQKTQGLRRFAFPLSLFQG LEKNVSVMTMI FKESSPSHPTRCHEDNVVVAVDSATNQVLHFQKTQGLRRFSFPLSLFQG LEKNVSVMTMIEKESSPSHPTRCHEDNVVVAVDSTTNRVLHFQKTQGLRRFSFPLSLFQG

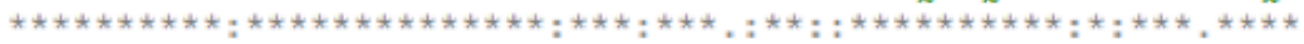
EIF2B5 gene : S447L $\checkmark$ CDRAEVKERVILKPHCVLTSQVVVGPDIILPEGSVISLHPPDAEEDEDDGQFSDDSGADQ CDRAEVKERVKLKPYCVLTSQVVVGPDITLPEGSVISLHPPDAEEDEDDGQFSDDSGADQ CDHAEVKEQVTLKPHCVLTSQVVVGPNITLPEGSVISLHPPDAEEDEDDGQFSDDSGVNQ CDNAEVKERVTLKPRCVLTSQVVVGPNIML PEGSVISLHPPDAEEDEDDGEFSDDSGTDQ CDNAEVKERVTLKPRSVLTSQVVVGPNITLPEGSVISLHPPDAEEDEDDGEFSDDSGADQ CDNAEVKERVTLKPRCVLTSQVVVGPNITLPEGSVISLHPPDAEEDEDDGEFSDDSGADQ CDNAEVKENVTLKPHCVLTSQVVVGPDIML PEGSVISLHPPDAEDDEDDGQFSDDSGADQ CDNAEVKEQVTLKPRCVLTSQVVVGPDITLPEGSVISLHPPDAEEDEDDGQFSDDSGANQ

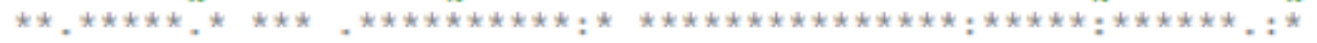

\section{EIF2B2 gene:V85E}

LMEI IRKEGRRMTAAQPSETTVGNMIRRVLKI IREEYARSRGSSEEADQQESLHKLLTSG LMDLIRREGRRMTAAQPSETTVGNMVRRVLKI IREEYGRLHGRSDESDQQESLHKLLTSG LMDLIRREGRRMTAAQPSETTVGMMVRVLKI IREEYGRLHGRSDESDQQESLHKLITSG LMELIRREGRRMTAAQPSETTVGNMVRRVLKI IREEYGRLHGRSDESDQQESLHKLLTSG LMELIRREGRRMMAAQPSETTVGIMVRRVLKI IREEYGRLHGRSDESDQQESLHKLLTSG LMELIRREGRRMTAAQPSETTVGNMVRRVLKI IREEYGRLHGRSDESDQQESLHKLLTSG LMELIRREGRRMTAAQPSETTVGNMVRRVLKI IREEYGRLHGRSDESDQQESLHKLITSG LMELIRREGRRMTAAQPSETTVGNMVRRVLRI IREEYGRLHGRSDESDQQESIHKLITSG

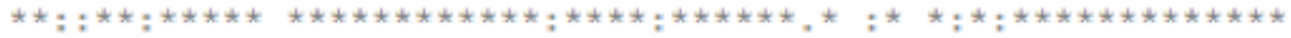

\section{Figure 6}

Conservation analysis of mutations using Clustal Omega software.Alignment of the region surrounding the mutations in human EIF2B5 and EIF2B2 with the corresponding sequences in other species. Residues that are conserved in at least 8 species are shown using the Clustal Omega software (http://www.ebi.ac.uk/Tools/msa/clustalo/). 


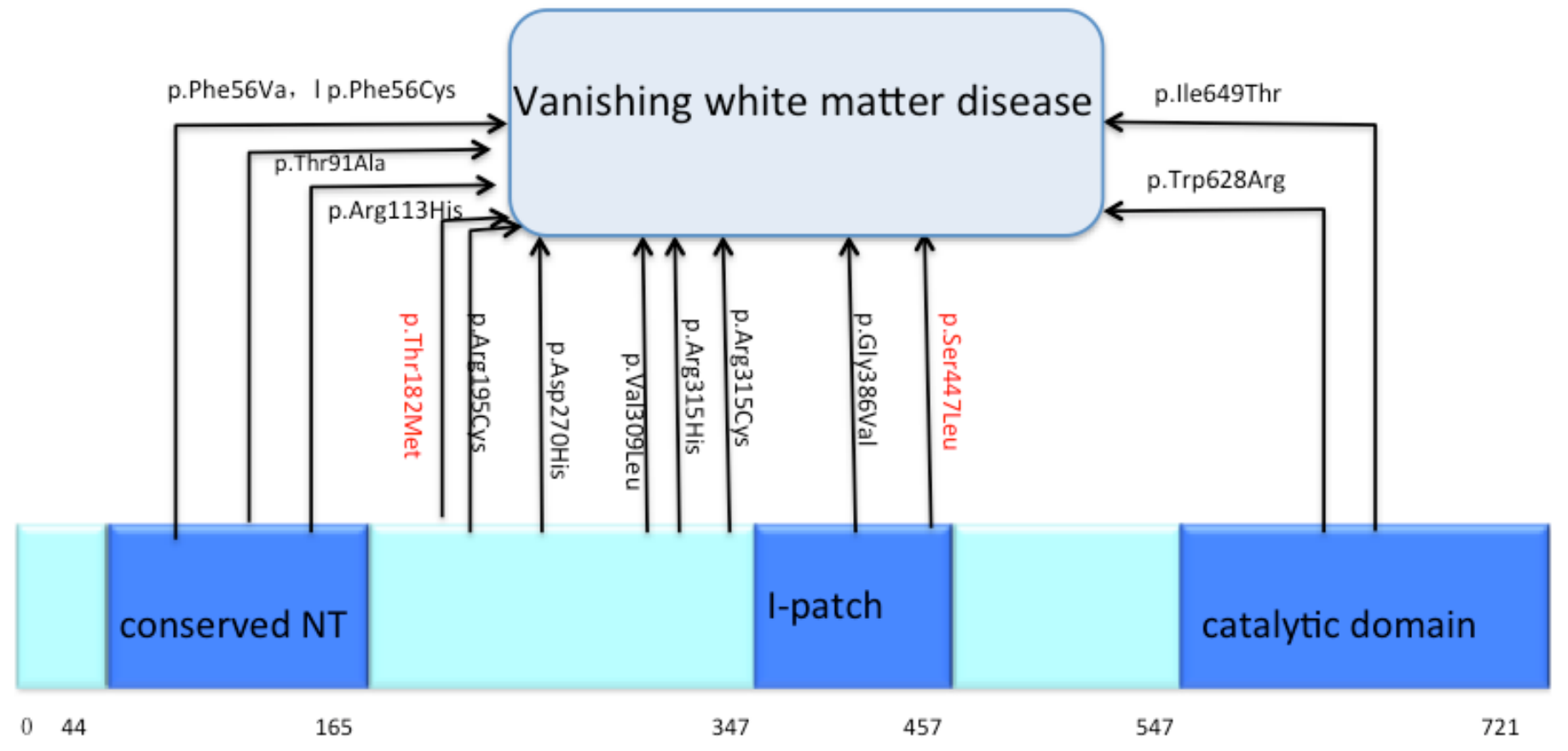

Figure 7

EIF2B5 protein domain and distribution of fourteen pathogenic missense mutation sites causing vanishing white matter disease on the domain. p.T182M and p.S447L are missense mutations we found in our case, and p.S447L is a newly discovered mutation that has not been reported before.

\section{Supplementary Files}

This is a list of supplementary files associated with this preprint. Click to download.

- CAREchecklistEnglish2020.1.28.pdf 